ARTICLE

https://doi.org/10.1038/s41467-019-11794-6

\title{
Isolating contiguous Pt atoms and forming Pt-Zn intermetallic nanoparticles to regulate selectivity in 4-nitrophenylacetylene hydrogenation
}

\author{
Aijuan Han ${ }^{1,2,7}$, Jian Zhang (10) 2,7, Wenming Sun (10 3,7, Wenxing Chen ${ }^{2,4}$, Shaolong Zhang ${ }^{2}$, Yunhu Han ${ }^{2}$, \\ Quanchen Feng ${ }^{2}$, Lirong Zheng ${ }^{5}$, Lin $\mathrm{Gu}^{6}$, Chen Chen², Qing Peng ${ }^{2}$, Dingsheng Wang $\mathbb{C}^{2} \&$ Yadong $\mathrm{Li}^{2}$
}

Noble metals play a momentous role in heterogeneous catalysis but still face a huge challenge in selectivity control. Herein, we report isolating contiguous Pt atoms and forming $\mathrm{Pt}-\mathrm{Zn}$ intermetallic nanoparticles as an effective strategy to optimize the selectivity of Pt catalysts. Contiguous Pt atoms are isolated into single atoms and Pt-Zn intermetallic nanoparticles are formed which are supported on hollow nitrogen-doped carbon nanotubes (PtZn/HNCNT), as confirmed by aberration-corrected high-resolution transmission electron microscopy and X-ray absorption spectrometry measurements. Interestingly, this PtZn/HNCNT catalyst promotes the hydrogenation of 4-nitrophenylacetylene to 4-aminophenylacetylene with a much higher conversion ( >99\%) and selectivity (99\%) than the comparison samples with $\mathrm{Pt}$ isolated-single-atomic-sites (Pt/HNCNT) and Pt nanoparticles (Pt/CN). Further density functional theory (DFT) calculations disclose that the positive $\mathrm{Zn}$ atoms assist the adsorption of nitro group and $\mathrm{Pt}-\mathrm{Zn}$ intermetallic nanoparticles facilitate the hydrogenation on nitro group kinetically.

\footnotetext{
${ }^{1}$ State Key Laboratory of Chemical Resource Engineering, Beijing University of Chemical Technology, Beijing 100029, China. ${ }^{2}$ Department of Chemistry, Tsinghua University, Beijing 100084, China. ${ }^{3}$ College of Science, China Agricultural University, Beijing 100193, China. ${ }^{4}$ Beijing Key Laboratory of Construction Tailorable Advanced Functional Materials and Green Applications, School of Materials Science and Engineering, Beijing Institute of Technology, Beijing 100081, China. ${ }^{5}$ Beijing Synchrotron Radiation Facility, Institute of High Energy Physics, Chinese Academy of Sciences, Beijing 100049, China. ${ }^{6}$ Institute of Physics, Chinese Academy of Sciences, Beijing 100190, China. ${ }^{7}$ These authors contributed equally: Aijuan Han, Jian Zhang, Wenming Sun. Correspondence and requests for materials should be addressed to D.W. (email: wangdingsheng@mail.tsinghua.edu.cn)
} 
A lthough noble metals are widely applied as heterogeneous catalysts in industrial processes, they are generally less selective to obtain the desired products with high yields $^{1-4}$. Due to their specific electronic structure, traditional noble catalysts are usually highly active, but have great difficulty in discriminating competitive functional groups in many cases $^{5-15}$. For instance, Pt catalyzed selective hydrogenation of the nitro group in nitrophenylacetylenes to obtain aminophenylacetylenes is a vital process in the production of antitumor agent Erlotinib and intermediates of fluorescent labels ${ }^{16-18}$. However, conventional $\mathrm{Pt}$ catalysts usually hydrogenate the highly reductive alkynyl group and the nitro group simultaneously, leading to a poor selectivity of desired products. Hence, exploiting effective strategies to modulate the selectivity of such Pt catalysts has drawn extensive research attention.

Tuning the electronic structure of noble metals has been emerging as an efficient strategy to optimize their catalytic selectivity ${ }^{19-22}$. Through this strategy, the adsorption/desorption properties of the relevant reaction species can be changed and the catalytic reaction pathway may be altered to achieve the ideal catalytic performance. So far, several approaches have been developed to adjust the electronic structures of noble metals, such as using surface organic ligand modifiers ${ }^{23-25}$, and creating metal-support interactions ${ }^{26-31}$. Besides, the construction of intermetallic compounds (IMCs) represents another powerful and flexible tool in this respect ${ }^{32-44}$. Upon precise design, noble metal atoms might be isolated into single atom on intermetallic structures orderly with the insertion of an inert metal (IM). Moreover, the formed noble metal-IM (NM-IM) bonds might alter the adsorption/desorption ability of reaction species and the reaction pathway, leaving possibility of catalyzing the reaction in the desired direction. Yet, researches devoted to improve the catalytic selectivity of NMs by this isolating contiguous NM atoms and forming NM-IM intermetallic nanoparticles strategy are still scarce.

Herein, we report isolating contiguous Pt atoms and forming $\mathrm{Pt}-\mathrm{Zn}$ intermetallic nanoparticles effectively regulate the selectivity of Pt catalysts. PtZn IMCs supported on hollow nitrogen doped carbon nanotubes (PtZn/HNCNT) are synthesized by a sacrificial template method. Interestingly, this PtZn/HNCNT greatly increases the catalytic conversion (>99\%) and selectivity (99\%) in hydrogenation of 4-nitrophenylacetylene (4-NPA) to 4aminophenylacetylene (4-APA) with ammonia borane as the hydrogenation source, outperforming the samples with $\mathrm{Pt}$ isolated-single-atomic-sites ( $\mathrm{Pt} / \mathrm{HNCNT}$ ) and $\mathrm{Pt}$ nanoparticles $(\mathrm{Pt} / \mathrm{CN})$. Density functional theory (DFT) calculation reveals that the adsorption of nitro group is enhanced due to the $\mathrm{Zn}$ atoms, and the hydrogenation on nitro group is kinetically favored due to the $\mathrm{Pt}-\mathrm{Zn}$ intermetallic nanoparticles.

\section{Results}

Synthesis and characterization of the catalyst. PtZn/HNCNT was fabricated through a sacrificial template method (Fig. 1a). First, $\mathrm{ZnO}$ nanorods were coated with a thin layer of polydopamine. Second, $\mathrm{Pt}(\mathrm{OH})_{4}$ was loaded on the outlayer through a precipitation method. The as-prepared $\mathrm{Pt}(\mathrm{OH})_{4} / \mathrm{ZnO} @ P D A$ was then reduced by hydrogen at $800^{\circ} \mathrm{C}$ to form PtZn IMCs, which is the critical step in this strategy. In this step, the $\mathrm{ZnO}$ was reduced to zinc vapor, which assists the contiguous $\mathrm{Pt}$ atoms isolated by $\mathrm{Zn}$ atoms to form the PtZn IMCs at high temperature. Finally, an acid wash step was carried out to remove remaining $\mathrm{ZnO}$.

Electron microscope was applied to observe the sample morphologies in the synthetic process. A $\sim 20-30 \mathrm{~nm}$ thin layer of PDA was coated on the outside of $\mathrm{ZnO}$ nanorods after step 1 (Supplementary Fig. 1). No obvious nanoparticles existed in this layer after step 2, indicating the ultrasmall size of the $\mathrm{Pt}(\mathrm{OH})_{4}$ (Supplementary Fig. 2). After high-temperature treatment and acid wash, HNCNTs were formed with 2-6 nm nanoparticles supported on them (Fig. 1b-d and Supplementary Fig. 3). Aberration-corrected high-angle annular dark-field scanning transmission electron microscope (AC HAADF STEM) images were taken to measure the particle distribution (Supplementary Fig. 4), and the average particle size was $2.24 \mathrm{~nm}$. XRD was then performed to examine the phase composition of the samples (Supplementary Fig. 5). Only graphitic carbon peaks were observed because of the ultrasmall size of the PtZn particles. A sample with higher Pt loading (1\%) is prepared using the same method. The diffraction peaks in XRD (Supplementary Fig. 6) match well to that of PtZn (JCPDS No. 06-0604). To further testify the IMC nature of PtZn particles, AC HAADF STEM measurement was carried out. AC HAADF STEM of one PtZn nanoparticle exhibited clear bright dots, in agreement with the atomic arrangement of $(02 \overline{2})$ plane in the PtZn crystal structure (Fig. 1e). The lattice spacing of 0.221 and $0.203 \mathrm{~nm}$ matched interplane distances of (111) and (200) planes of intermetallic PtZn (P4/mmm) respectively (Fig. 1f). The perfect agreement testified the nanoparticle is PtZn IMC. More AC HAADF STEM images are taken to prove that the nanoparticles are PtZn IMC. As shown in Supplementary Fig. 7, the atomic arrangement of another nanoparticle could match well with the (010) plane of PtZn. Due to the different atomic density, the heavier Pt atoms (bright) can be distinguished from $\mathrm{Zn}$ atoms (darker). Therefore, we think that the PtZn IMCs instead of core-shell alloys are formed in the PtZn/HNCNT. Further energy-dispersive X-ray (EDX) elemental mapping (Fig. 1g) analysis exhibited the simultaneous presence and homogeneous dispersion of $\mathrm{Pt}$ and $\mathrm{Zn}$, indicating the uniform composition of the particle. For comparison, $\mathrm{Pt} / \mathrm{HNCNT}$ was also prepared in a similar procedure except reduced at a low temperature $\left(200^{\circ} \mathrm{C}\right)$, where only $\mathrm{Pt}$ component is present on the HNCNT support due to the absence of $\mathrm{Zn}$ vapor (its characterizations are shown in Supplementary Figs. 8-12). AC HAADF STEM image (Supplementary Fig. 12) shows only bright dots on the support, indicating that the $\mathrm{Pt}$ component exists in the form of isolated-single-atomic-sites. Pt/ $\mathrm{CN}$, prepared by a similar method using polydopamine nanospheres as the support and treated in $\mathrm{H}_{2} / \mathrm{Ar}$ atmosphere at $800^{\circ} \mathrm{C}$, contains obvious Pt nanoparticles, as deduced from XRD, TEM, and AC HAADF STEM (Supplementary Fig. 13). These results prove that $\mathrm{Pt}$ atoms tend to aggregates upon high temperature, and the presence of $\mathrm{ZnO}$ helps to isolate contiguous $\mathrm{Pt}$ atoms by forming $\mathrm{Pt}-\mathrm{Zn}$ bridge sites in the $\mathrm{PtZn}$ intermetallic nanoparticles. Using the similar method, PdZn/HNCNT with $\mathrm{PdZn}$ intermetallic nanoparticles supported on HNCNT was successfully obtained (its characterizations are shown in Supplementary Fig. 14), proving the generality of this strategy to synthesize IMCs.

To verify the different electronic structures between PtZn/ HNCNT and Pt/HNCNT, X-ray photoelectron spectroscopy (XPS) and X-ray absorption spectrometric (XAS) measurements were performed. Supplementary Fig. 15 shows the XPS analysis at Pt $4 f$. Compared with $\mathrm{Pt} / \mathrm{HNCNT}$, a shift to lower binding energy is observed for PtZn/HNCNT. The $\mathrm{Zn} 2 p$ peak of PtZn/HNCNT is shifted to higher binding energy than metallic zinc (Supplementary Fig. 16) ${ }^{45}$. These phenomena indicate the Pt electronic states are modified by $\mathrm{Zn}$ in $\mathrm{PtZn} / \mathrm{HNCNT}$ via electron transfer from $\mathrm{Zn}$ to $\mathrm{Pt}$ atoms. As seen from the normalized X-ray absorption near-edge structure (XANES) curves at the $\mathrm{Pt}_{\mathrm{L}_{3} \text {-edge }}$ (Fig. 2a), PtZn/HNCNT has lower energy of the adsorption edge $\left(\mathrm{E}_{0}\right)$ than Pt foil and Pt/HNCNT, suggesting the electron richness of $\mathrm{Pt}$ atoms in PtZn/HNCNT. In comparison with $\mathrm{Zn}$ foil, this 

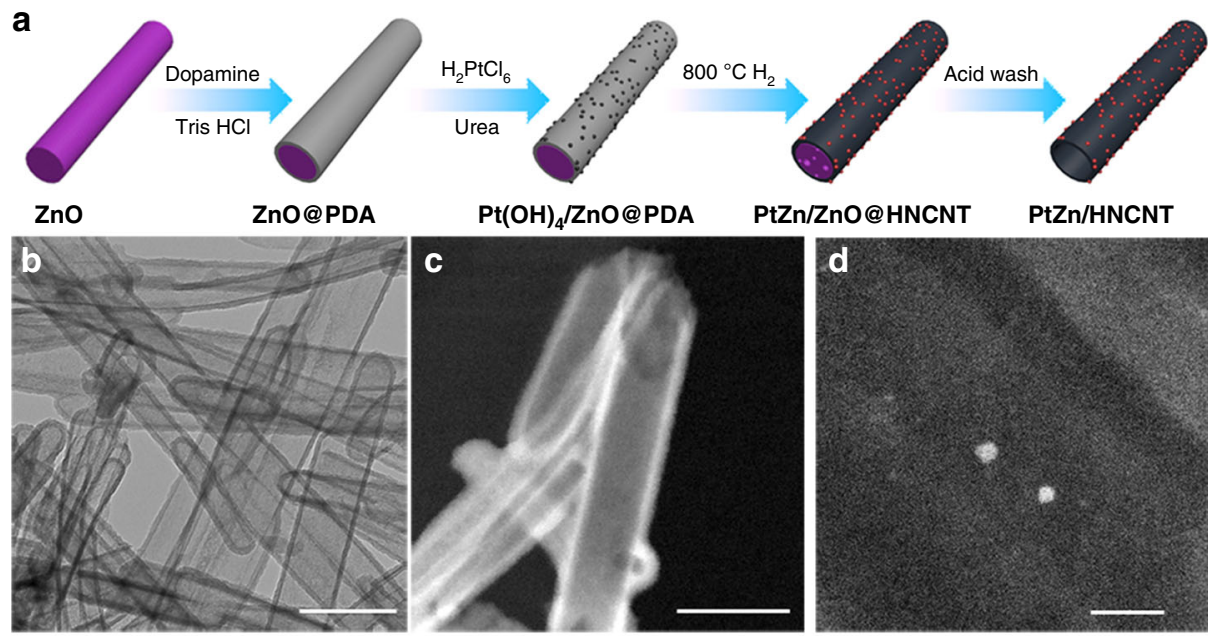

$\mathrm{Pt}(\mathrm{OH})_{4} / \mathrm{ZnO} @ \mathrm{PDA}$

PtZn/ZnO@HNCNT

PtZn/HNCNT
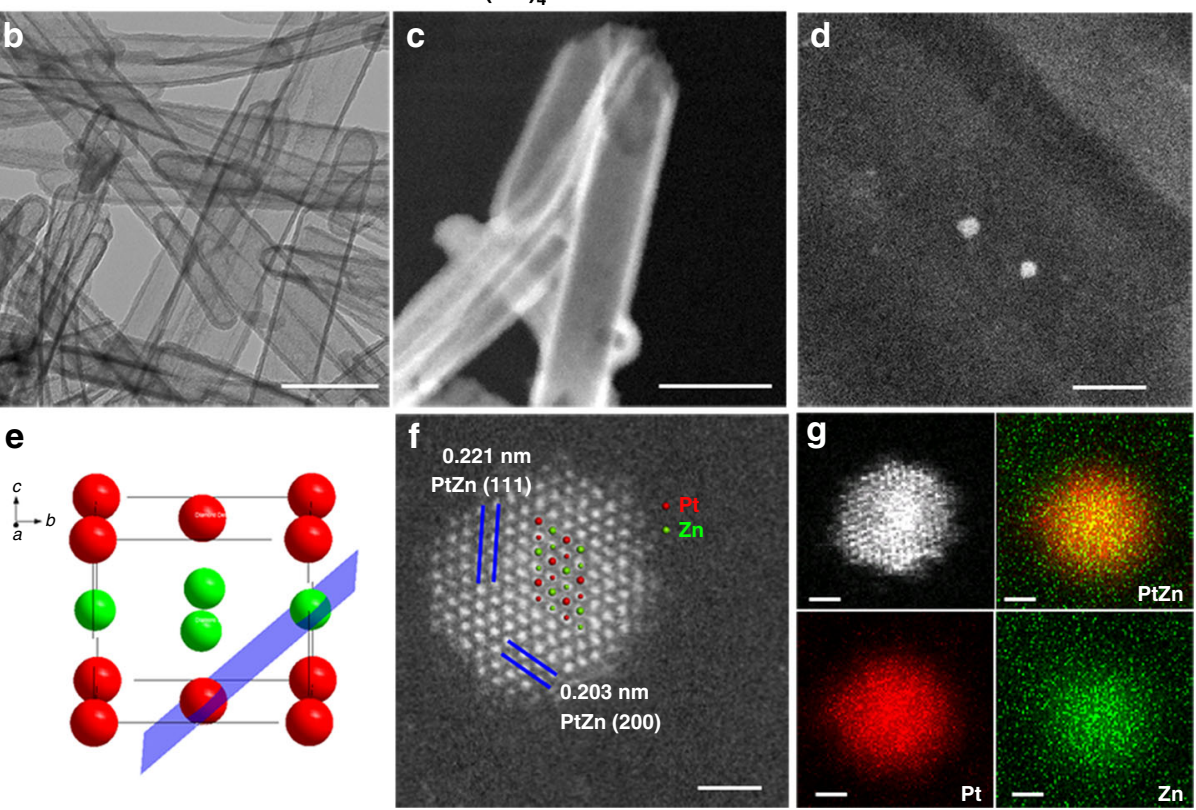

Fig. 1 Synthetic scheme and characterization of the catalyst. a Synthetic strategy of PtZn intermetallic nanoparticles supported on hollow nitrogen-doped carbon nanotubes (PtZn/HNCNT). b-d, Transmission electron microscope (TEM) (b), high angel annular dark field scanning TEM (HAADF STEM) (c), and aberration-corrected (AC) HAADF STEM (d) images of PtZn/HNCNT. Scale bar, $500 \mathrm{~nm}$ (b); $200 \mathrm{~nm}$ (c); $10 \mathrm{~nm}$ (d). e Crystal structure of PtZn intermetallic compound (IMC) (Pt: red; Zn: green). f, g AC HAADF STEM (f) image and elemental mappings (g) of a PtZn IMC nanoparticle. Scale bar, $1 \mathrm{~nm}$

material exhibits higher height of white line $\left(H_{\mathrm{w}}\right)$ and $\mathrm{E}_{0}$ at the $\mathrm{Zn}$ K-edge (Fig. 2b). It demonstrates the electron deficiency at the Zn atoms in PtZn/HNCNT, in accordance with the result of $\mathrm{Pt} \mathrm{L}_{3^{-}}$ edge XANES spectra. Furthermore, the corresponding Fourier transform of extended X-ray absorption fine structure (EXAFS) oscillations (Supplementary Figs. 17 and 18) are performed at $\mathrm{Pt}$ $\mathrm{L}_{3}$-edge and $\mathrm{Zn} \mathrm{K}$-edge. The first nearest-coordination peak of $\mathrm{PtZn} / \mathrm{HNCNT}$ displayed a slight shift in $\mathrm{R}$ space than that of $\mathrm{Pt}$ foil at $\mathrm{Pt}_{3}$-edge (Fig. 2c), while a slight shift in reverse direction was also observed at $\mathrm{Zn} \mathrm{K}$-edge (Fig. 2d), indicating the mild change of atomic distance. Pt-Pt bonds at around $2.4 \AA$ were obviously observed in $\mathrm{Pt} / \mathrm{CN}$, while only a peak at $1.6 \AA$ in the first shell was observed in Pt/HNCNT (Fig. 2c). EXAFS fitting were further carried out to obtain quantitative structural configuration of Pt in the three samples (Supplementary Fig. 19 and Table S1). The average coordination number of Pt/HNCNT is 3.8, indicating a Pt- $\mathrm{N}_{4}$ structure. The experimental curves of $\mathrm{Pt} / \mathrm{CN}$ and PtZn/HNCNT could fit well with that of Pt metals and $\mathrm{PtZn}$ IMC respectively, furthering confirming that the $\mathrm{Pt}$ dispersed as nanoparticles and isolated-single-atomic-sites in $\mathrm{Pt} / \mathrm{CN}$ and $\mathrm{Pt} / \mathrm{HNCNT}$, respectively.

CO adsorption was carried out to study the dispersion and electronic structures of Pt phase (Supplementary Fig. 20). There is a red shift of the CO stretching frequency for PtZn/HNCNT compared to $\mathrm{Pt} / \mathrm{CN}$ (from 2083 to $2053 \mathrm{~cm}^{-1}$ ), indicating that the electron density is increased for PtZn/HNCNT due to the presence of zinc ${ }^{46-51}$, in consistent with the observation of $\mathrm{Pt}$ XPS. Pt/HNCNT demonstrates a peak at $2143 \mathrm{~cm}^{-1}$, further confirming that the $\mathrm{Pt}$ component in this sample is dispersed as isolated-single-atomic-sites ${ }^{52}$.
Catalytic performance for selective hydrogenation. Hydrogenation of 4-NPA (1) was then exploited as a model reaction to investigate the catalytic performance of PtZn/HNCNT. Since there are two easily reducible groups, nitro group and alkynyl group, the hydrogenation reaction has two possible pathways (Fig. 3a). To our delight, the PtZn/HNCNT exhibited a high hydrogenation selectivity to nitro functional group, producing 4APA (2a). The conversion was $100 \%$ and the selectivity was $99 \%$ within $4 \mathrm{~h}$ using ammonia borane as the hydrogenation source (Fig. 3b). Trace amount of further hydrogenation product 4aminostyrene (2b) was also detected. The $1 \mathrm{H}$ NMR and $13 \mathrm{C}$ NMR further confirmed the product is 4-aminophenylacetylene (Supplementary Figs. 21 and 22) and the mass recovery yield of 2b is $93 \%$. The kinetic profile was followed and shown in Supplementary Fig. 23. The conversion increased fast in the first $2 \mathrm{~h}$ while the selectivity kept $99 \%$ to 2 a. Different reaction conditions were also studied and shown in Supplementary Table 2. The highest selectivity was obtained at $40^{\circ} \mathrm{C}$. Since the reaction was carried out in the pressure tube, a larger volume of tube was used to reduce the pressure during the reaction. However, a decreased selectivity was observed. In comparison, the $\mathrm{Zn}$-free catalyst $\mathrm{Pt} /$ HNCNT was also applied into such reaction, but exhibited rather different performance as compared to PtZn/HNCNT. Nearly $30 \%$ conversion was observed after $4 \mathrm{~h}$ while a complex mixture containing $5 \%$ of $\mathbf{2 b}, 47 \%$ of 4 -nitrostyrene (3a), $6 \%$ of 4 nitroethylbenzene (3b), and $42 \%$ of 4 -nitroacetophenone (3c) (generated from the oxidation of $\mathbf{3 a}$ by the remaining air during the reaction process) was obtained. Nearly no reaction occurred in the absence of Pt (i.e., HNCNT, its characterizations are shown in Supplementary Figs. 24 and 25), proving that the active species 

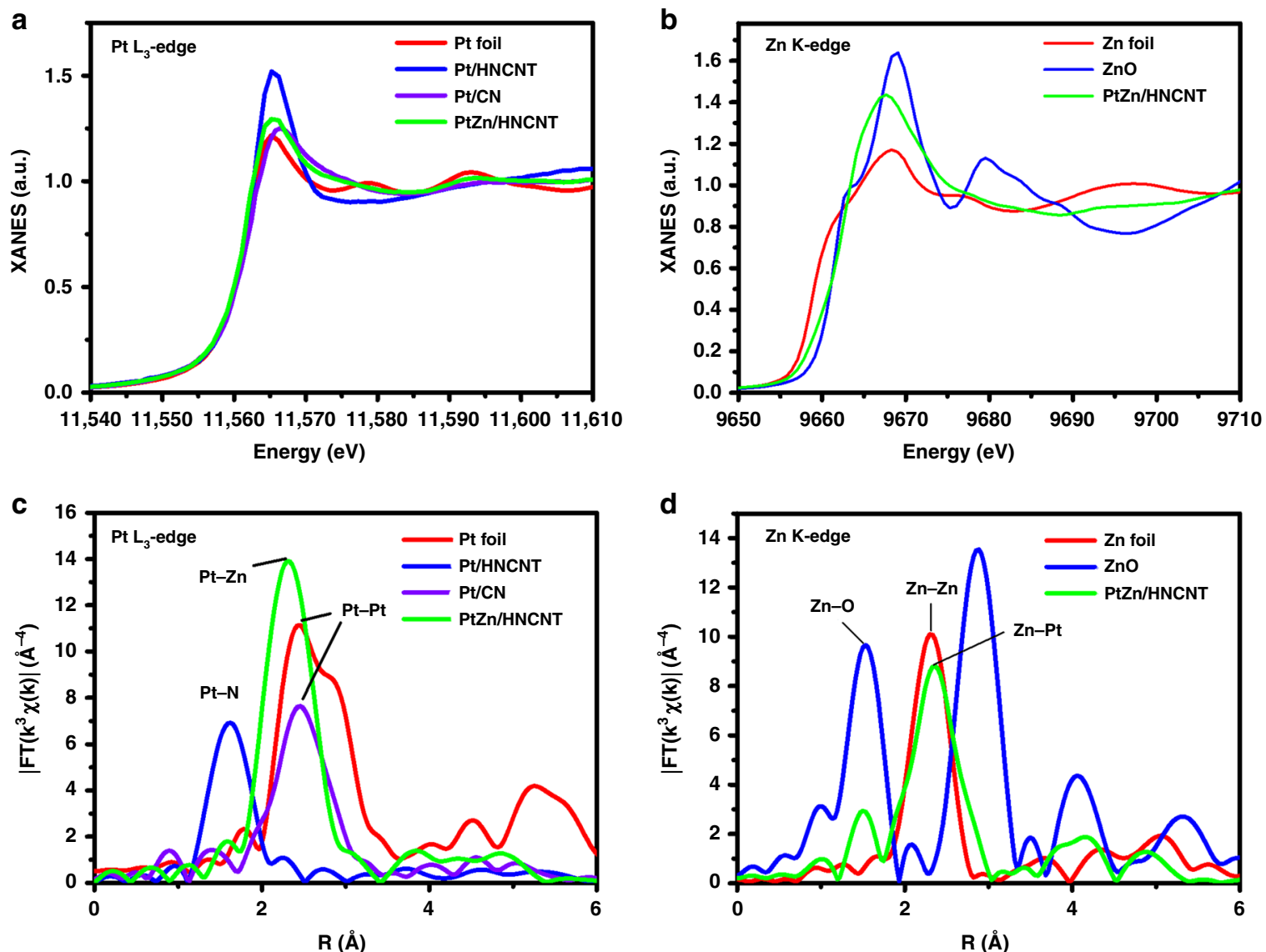

Fig. 2 X-ray absorption spectroscopy characterization of the catalysts. a, c The normalized X-ray absorption near-edge structure (XANES) spectra (a) and Fourier transform extended X-ray absorption fine structure (FT-EXAFS) (c) at the Pt $\mathrm{L}_{3}$-edge of the PtZn/HNCNT, Pt isolated-single-atomic-site supported on hollow nitrogen-doped carbon nanotubes (Pt/HNCNT), Pt nanoparticles supported on nitrogen-doped carbon nanospheres (Pt/CN) and Pt foil. b, $\mathbf{d}$ The normalized XANES spectra (b) and FT-EXAFS (d) at the Zn K-edge of the PtZn/HNCNT, ZnO and Zn foil

are the Pt metals. $\mathrm{Pt} / \mathrm{CN}$, which is prepared without using $\mathrm{ZnO}$ and thus bearing Pt nanoparticles supported on $\mathrm{CN}$ support, was also compared. It exhibited similar selectivity to that of $\mathrm{Pt} /$ HNCNT, with $39.7 \%$ selectivity to $3 \mathbf{a}$. To compare the potential difference in selectivity of those catalysts, the selectivity was compared at similar conversion level (Supplementary Fig. 26). PtZn/HNCNT exhibited extremely high selectivity to 2 a compared to the Pt/HNCNT and Pt/CN samples. Obviously, PtZn/ HNCNT catalyzed the hydrogenation of 4-NPA in different routes to $\mathrm{Pt} / \mathrm{HNCNT}$ and $\mathrm{Pt} / \mathrm{CN}$. With isolating contiguous $\mathrm{Pt}$ and forming $\mathrm{Pt}-\mathrm{Zn}$ bridge site, the PtZn/HNCNT can distinctively hydrogenate the nitro group in 4-NPA, achieving a high selectivity of $2 \mathrm{a}$. The catalytic performance of $\mathrm{ZnO}$ is also tested. It showed $51 \%$ conversion after $4 \mathrm{~h}$, with $33.9 \%$ to $3 \mathrm{a}$. $\mathrm{ZnO}$ exhibits preferential hydrogenation to the alkynyl group. Since PtZn/ HNCNT demonstrates preferential hydrogenation to the nitro group, it further excluded that zinc is the real active phase in the PtZn/HNCNT catalyst. In this study, PtZn/HNCNT can realize this process with a high selectivity in rather mild condition, which might promote the development of the selective hydrogenation of 4-NPA. Nitrobenzenes with electron donating and withdrawing groups were also used as the substrates (Supplementary Table 3). PtZn/HNCNT showed high selectivity for functionalized nitrobenzenes with electron donating or withdrawing groups in different positions. It indicated the outstanding performance of $\mathrm{PtZn} /$ HNCNT in selective hydrogenation of nitrobenzenes. Besides, the stability of the catalyst was tested. After reaction, there was nearly no change in the metal content $(0.82 \%$ before and $0.80 \%$ after reaction), XRD, XPS, XAS, $\mathrm{N}_{2}$ sorption, and in situ Fouriertransform infrared spectroscopy of CO chemisorptions for PtZn/ HNCNT (Supplementary Figs. 27-32). No obvious decrease in the catalytic performance was observed (Supplementary Fig. 33), suggesting the robust nature of the PtZn/HNCNT.

DFT calculations of hydrogenation on the catalysts. To gain more insight into the high selectivity reason of $\mathrm{PtZn} / \mathrm{HNCNT}$, DFT calculations were utilized to distinguish the adsorption energies and hydrogenation energy barriers of 4-NPA on PtZn IMCs and Pt metals. The optimized geometry of 4-NPA@Pt(111) is shown in Supplementary Fig. 34. The distances between nitro group, aromatic ring, alkynyl group, and metal surface decrease gradually. The configuration which nitro group is most close to $\mathrm{Pt}$ surface was also considered (Supplementary Fig. 34), however, this configuration is $94 \mathrm{~kJ} \mathrm{~mol}^{-1}$ less stable. For $\mathrm{PtZn}(02 \overline{2})$ surface, the shortest distance between Pt atom in the same layer is $4.026 \AA$, which is significantly longer than it in $\operatorname{Pt}(111)$ surface $(2.804 \AA)$. Meanwhile the shortest distance between $\mathrm{Pt}$ and $\mathrm{Zn}$ in the same layer is $2.639 \AA$. In this sense, the Pt atom on the surface was isolated partly by two nearest-neighboring $\mathrm{Zn}$ atoms. The difference in electronegativity between $\mathrm{Pt}$ and $\mathrm{Zn}$ implies that the $\mathrm{Pt}$ atoms and $\mathrm{Zn}$ atoms in PtZn are negatively charged and positively charged, respectively, which is confirmed by the calculated charge population. As shown in Supplementary Fig. 35, maintaining more carbon atoms in both aromatic ring and alkynyl group close to $\mathrm{Pt}$ atoms and oxygen atoms in nitro close to $\mathrm{Zn}$ could make the configuration more stable. The adsorption 

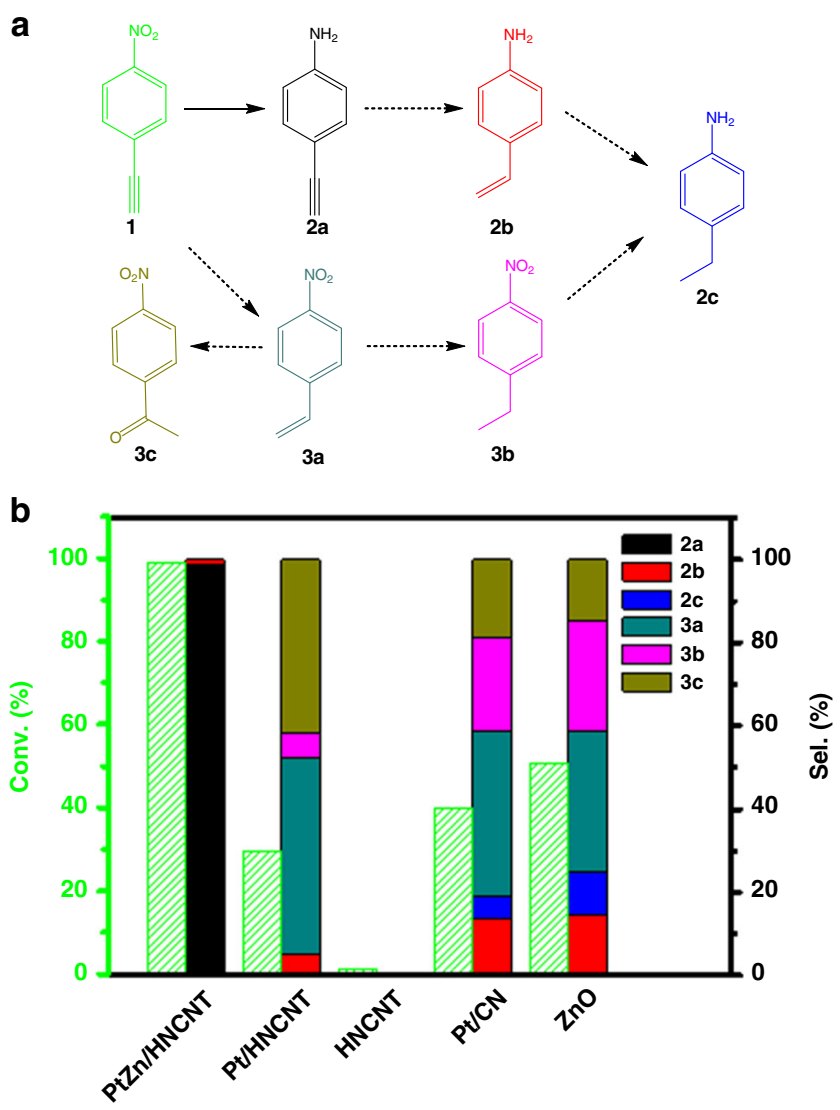

Fig. 3 Catalytic performance of the catalysts. a Reaction pathway of the hydrogenation of 4-nitrophenylacetylene (4-NPA). b The catalytic performance of PtZn/HNCNT, Pt/HNCNT, hollow nitrogen-doped carbon nanotube (HNCNT), Pt/CN, and $\mathrm{ZnO}$

configurations of 4-NPA at the $\mathrm{Pt}$ isolated-single-atomic-site (Pt-N-C) were also calculated and found that the one with the aromatic ring adsorbed on the $\mathrm{Pt}-\mathrm{N}_{4}$ is more stable, but the difference between different modes was not big (Supplementary Fig. 36).

Hydrogenation processes could potentially occur on both nitro and alkynyl groups. To reveal the chemoselective hydrogenation process, the first elementary reaction steps on both groups were investigated. Hydrogen atom prefers to adsorb on the fcc-hollow site on Pt surface (Supplementary Fig. 37). Over Pt(111) surface, the energy barriers in the first step for hydrogenation on nitro group and alkynyl group are 239 and $117 \mathrm{~kJ} \mathrm{~mol}^{-1}$ (Fig. 4a), respectively, indicating that the hydrogenation process on nitro group is unfavorable kinetically. The thermochemistry-only potential energy pathways were constructed (Supplementary Figs. 38-47). In the first two hydrogenation steps, the alkynyl group is the favorable target, followed by the formation of vinyl group (intermediate 3a in Fig. 3). The vinyl group does not achieve ethyl till the tenth hydrogenation step, meanwhile, the last hydrogenation step is endothermic. The long-lasting unsaturated $\mathrm{C}_{2} \mathrm{H}_{4}$-group should be the primitive intermediate for the formation of $\mathrm{CH}_{3}-\mathrm{CO}$ $\mathrm{C}_{6} \mathrm{H}_{4}-\mathrm{NO}_{2}$ (3c in Fig. 3) and $\mathrm{CH}_{3}-\mathrm{CH}_{2}-\mathrm{C}_{6} \mathrm{H}_{4}-\mathrm{NO}_{2}$ (3b in Fig. 3). Over PtZn (02) 2 ) surface, hydrogen atom prefers to adsorb on the $\mathrm{Pt}-\mathrm{Zn}$ bridge site (Supplementary Fig. 48). The relative vertical distances between $\mathrm{H}^{\star}$ and its hydrogenation target atom (oxygen in nitro group or carbon atom in alkynyl group) are comparable (0.897 vs. $0.978 \AA)$. For 4-NPA@PtZn $(02 \overline{2})$, hydrogenation on nitro group is along the hydrogen atom diffusion path from $\mathrm{Pt}-\mathrm{Zn}$ (bridge) to $\mathrm{Zn}-\mathrm{Pt}$ (bridge) (along $\langle 011\rangle$ direction, Supplementary

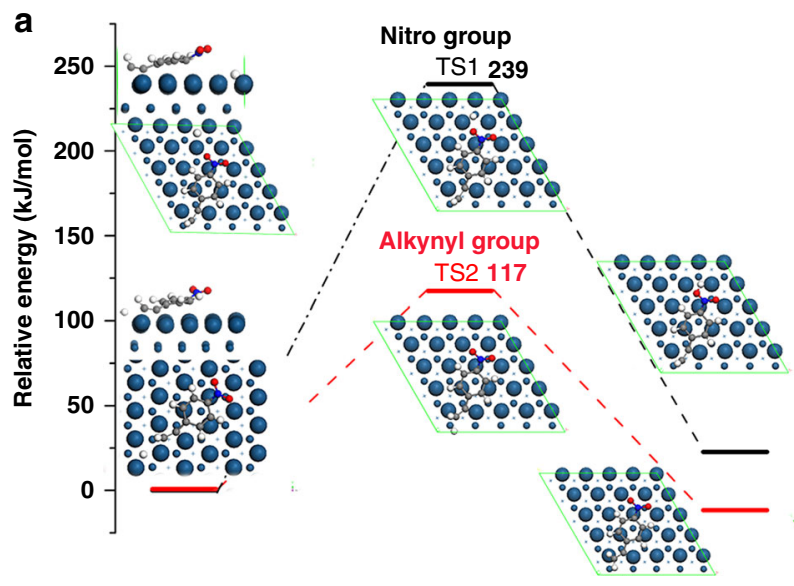

b

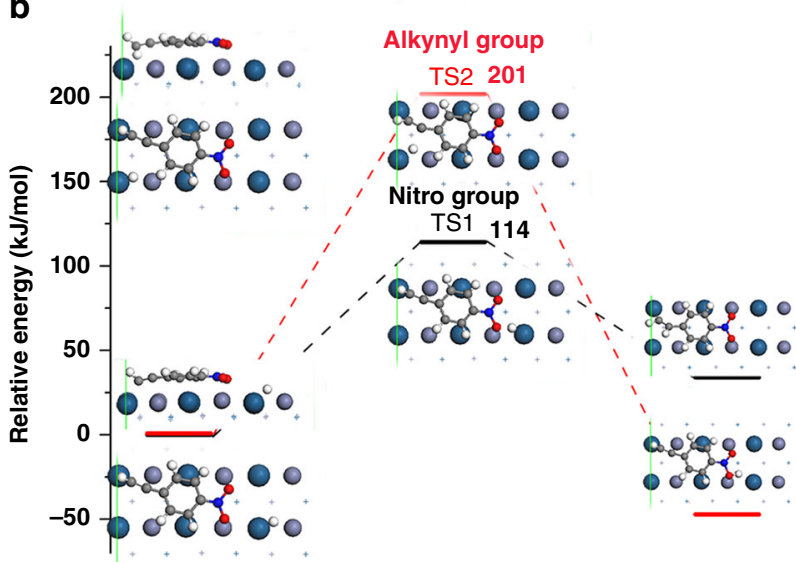

Fig. 4 DFT calculations of 4-nitrophenylacetylene over the catalysts. $\mathbf{a}, \mathbf{b}$ The first elementary hydrogenation reaction steps on both nitro and alkynyl groups over Pt(111) (a) and PtZn(02 2 ) (b) surface. Numbers labeled indicate the barriers of elementary steps (unit: $\mathrm{kJ} \mathrm{mol}^{-1}$ )

Fig. 49). The diffusion barrier is $29 \mathrm{~kJ} \mathrm{~mol}^{-1}$. Meanwhile, the hydrogenation on alkynyl group is along $\langle 100\rangle$ direction. Hydrogen diffusion along this path is unfavorable (diffusion barrier is $143 \mathrm{~kJ} \mathrm{~mol}^{-1}$ ). Interestingly, the energy barriers in the first step for hydrogenation on nitro group and alkynyl group are 114 and $201 \mathrm{~kJ} \mathrm{~mol}^{-1}$ (Fig. 4b), respectively, indicating that the hydrogenation process on alkynyl group is unfavorable kinetically. Based on the direct hydrogenation path nitro group, the barriers of first hydrogenation step on alkynyl group for $\mathrm{CH}-\mathrm{C}-\mathrm{C}_{6} \mathrm{H}_{4}-\mathrm{NOOH}$, $\mathrm{CH}-\mathrm{C}-\mathrm{C}_{6} \mathrm{H}_{4}-\mathrm{NO}, \quad \mathrm{CH}-\mathrm{C}-\mathrm{C}_{6} \mathrm{H}_{4}-\mathrm{NHO}, \mathrm{CH}-\mathrm{C}-\mathrm{C}_{6} \mathrm{H}_{4}-\mathrm{N}, \quad \mathrm{CH}-\mathrm{C}-$ $\mathrm{C}_{6} \mathrm{H}_{4}-\mathrm{NH}$, and $\mathrm{CH}-\mathrm{C}-\mathrm{C}_{6} \mathrm{H}_{4}-\mathrm{NH}_{2}$ on IMC surface were also calculated (Supplementary Figs. 50-59). We can find that every reaction needs to climb over a barrier higher than $200 \mathrm{~kJ} \mathrm{~mol}^{-1}$, which is difficult to trigger the subsequent hydrogenation reactions on the alkynyl group. We considered that the such high reaction barrier on alkynyl part is the essential reason for the high selectivity on PtZn surface.

\section{Discussion}

In conclusion, we have found that the selectivity of Pt catalysts can be effectively tuned by isolating Pt contiguous atoms and forming $\mathrm{Pt}-\mathrm{Zn}$ intermetallic nanoparticles. Notably, the PtZn/ HNCNT exhibits superior catalytic selectivity $(99 \%)$ of the hydrogenation of 4-NPA to 4-APA in comparison to Pt/HNCNT. DFT calculations reveal that the existence of $\mathrm{Zn}$ atoms increases the adsorption of nitro groups, and $\mathrm{Pt}-\mathrm{Zn}$ intermetallic nanoparticles promote the hydrogen atom diffusion path. This work 
provides a feasible strategy to tune the selectivity of NMs to achieve the desired products.

\section{Methods}

Synthesis of ZnO@PDA. ZnO nanorods were synthesized by a reported method $^{53}$. Totally, $0.1 \mathrm{~g} \mathrm{ZnO}$ nanorods were dispersed in $20 \mathrm{ml}$ Tris buffer solution ( $10 \mathrm{mM}, \mathrm{pH} 8.5$ ) by ultrasonication. Then $1 \mathrm{ml}$ dopamine hydrochloride $(100 \mathrm{mg}$ ) solution was added to the $\mathrm{ZnO}$ suspension while stirring. The suspension was allowed to stir for $12 \mathrm{~h}$ at room temperature. The resulting products were washed by deionized water and ethanol three times and collected by centrifugation. After dried at $80^{\circ} \mathrm{C}$ in an oven, the desired $\mathrm{ZnO@PDA} \mathrm{was} \mathrm{obtained.}$

Synthesis of Pt(OH) $\mathbf{4} / \mathbf{Z n O @ P D A}$. A total of $22.5 \mathrm{mg}$ of urea was added in $100 \mathrm{ml}$ of deionized water. Then, $200 \mathrm{mg}$ of $\mathrm{ZnO} @ \mathrm{PDA}$ was added into the solution and ultrasonicated for $30 \mathrm{~min}$. Totally, $10.5 \mu \mathrm{L}$ of $0.1 \mathrm{~g} \mathrm{ml}^{-1} \mathrm{H}_{2} \mathrm{PtCl}_{6}$ aqueous solution was added and stirred for $3 \mathrm{~h}$ at room temperature. The mixture was then heated up to $90^{\circ} \mathrm{C}$ for $12 \mathrm{~h}$. The products were centrifuged and washed with deionized water three times. After drying in an oven at $80^{\circ} \mathrm{C}$ overnight, $\mathrm{Pt}(\mathrm{OH})_{4} / \mathrm{ZnO} @ P D A$ was obtained.

Synthesis of PtZn/HNCNT. The obtained $\mathrm{Pt}(\mathrm{OH})_{4} / \mathrm{ZnO} @ \mathrm{PDA}$ was treated at $800{ }^{\circ} \mathrm{C}$ in a home-made porcelain boat with a cap under $\mathrm{H}_{2}$ (5\% in $\mathrm{Ar}$ ) atmosphere for $1 \mathrm{~h}$ in a tube furnace at a ramp rate of $5^{\circ} \mathrm{C} \mathrm{min}-1$. After cooling down to room temperature naturally, the heat treated samples were washed in $5 \mathrm{M} \mathrm{HCl}$ for $12 \mathrm{~h}$. Finally, the resulting product PtZn/HNCNT was obtained after the samples were centrifuged, washed with deionized water several times, and dried at $80^{\circ} \mathrm{C}$ in an oven.

Catalytic test for 4-nitrophenylacetylene hydrogenation. The selective hydrogenation of 4-nitrophenylacetylene (4-NPA) of the catalysts was tested in a $15 \mathrm{ml}$ pressure bottle with $2 \mathrm{mg}$ of the catalyst, $73.6 \mathrm{mg}(0.5 \mathrm{mmol})$ of $4-\mathrm{NPA}, 92.6 \mathrm{mg}$ ( $3 \mathrm{mmol}$ ) of ammonia borane, $0.1 \mathrm{ml}$ distilled water, and $4.9 \mathrm{ml}$ ethanol. The reaction was performed at $40^{\circ} \mathrm{C}$ for $4 \mathrm{~h}$. Liquid samples was analyzed by gas chromatography with a Thermo Finnigan chromatograph equipped with a flame ionization detector and a DB-WAX capillary column (J\&W, $30 \mathrm{~m}, 0.25 \mathrm{~mm}$ i.d.) with nitrogen as the carrier gas.

Characterizations. Transmission electron microscopy (TEM) images were taken on a Hitachi HT7700 transmission electron microscope working at $100 \mathrm{kV}$. The high-resolution TEM, HAADF-STEM images, and the corresponding EDX mapping were recorded by a JEOL JEM-2100F high resolution TEM operating at $200 \mathrm{kV}$. Aberration-corrected HAADF-STEM images are taken on a JEOL JEMARM200F TEM/STEM with a spherical aberration corrector working at $300 \mathrm{kV}$. Powder X-ray diffraction patterns were measured with a Bruker D8 with Cu Ka radiation $(\lambda=1.5406 \AA)$. The XPS was measured ex situ by a PHI Quantera SXM system under $3.1 \times 10^{-8} \mathrm{~Pa}$ using $\mathrm{Al}^{+}$radiation at room temperature. The binding energies were calibrated by referring $\mathrm{C} 1 s$ peak to $284.8 \mathrm{eV}$. The metal content was determined by inductively coupled plasma optical emission spectroscopy (ICPOES) on Thermo Fisher IRIS Intrepid II.

\section{Data availability}

The data that support the findings of this study are available from the corresponding author upon request.

Received: 31 August 2018 Accepted: 5 August 2019

Published online: 22 August 2019

\section{References}

1. Liu, L. \& Corma, A. Metal catalysts for heterogeneous catalysis: from single atoms to nanoclusters and nanoparticles. Chem. Rev. 118, 4981-5079 (2018).

2. Quan, Z., Wang, Y. \& Fang, J. High-index faceted noble metal nanocrystals. Acc. Chem. Res. 46, 191-202 (2013).

3. Shoaib, A. et al. Noble metal nanoclusters and their in situ calcination to nanocrystals: Precise control of their size and interface with $\mathrm{TiO}_{2}$ nanosheets and their versatile catalysis applications. Nano Res. 9, 1763-1774 (2016).

4. Liu, J., Krajangsri, S., Yang, J., Li, J.-Q. \& Andersson, P. G. Iridium-catalysed asymmetric hydrogenation of allylic alcohols via dynamic kinetic resolution. Nat. Catal. 1, 438-443 (2018).

5. Mao, J. et al. Rational control of the selectivity of a ruthenium catalyst for hydrogenation of 4-nitrostyrene by strain regulation. Angew. Chem. 129, 12133-12137 (2017).

6. Feng, Q. et al. Isolated single-atom pd sites in intermetallic nanostructures: high catalytic selectivity for semihydrogenation of alkynes. J. Am. Chem. Soc. 139, 7294-7301 (2017)
7. Wei, H. et al. FeOx-supported platinum single-atom and pseudo-single-atom catalysts for chemoselective hydrogenation of functionalized nitroarenes. Nat. Commun. 5, 5634 (2014).

8. Liu, L. et al. Generation of subnanometric platinum with high stability during transformation of a 2D zeolite into 3D. Nat. Mater. 16, 132-138 (2016).

9. Imaoka, T. et al. Platinum clusters with precise numbers of atoms for preparative-scale catalysis. Nat. Commun. 8, 688 (2017).

10. Lucci, F. R. et al. Selective hydrogenation of 1,3-butadiene on platinum-copper alloys at the single-atom limit. Nat. Commun. 6, 8550 (2015)

11. Vilé, G., Almora-Barrios, N., López, N. \& Pérez-Ramírez, J. Structure and reactivity of supported hybrid platinum nanoparticles for the flow hydrogenation of functionalized nitroaromatics. ACS Catal. 5, 3767-3778 (2015).

12. Iwasa, N., Mayanagi, T., Ogawa, N., Sakata, K. \& Takezawa, N. New catalytic functions of Pd-Zn, Pd-Ga, Pd-In, Pt-Zn, Pt-Ga and Pt-In alloys in the conversions of methanol. Catal. Lett. 54, 119-123 (1998).

13. Cuenya, B. R. \& Behafarid, F. Nanocatalysis: size- and shape-dependent chemisorption and catalytic reactivity. Surf. Sci. Rep. 70, 135-187 (2015).

14. Moliner, M. Gabay et al. Reversible transformation of Pt nanoparticles into single atoms inside high-silica chabazite zeolite. J. Am. Chem. Soc. 138, 15743-15750 (2016).

15. Vilé, G., Richard-Bildstein, S., Lhuillery, A. \& Rueedi, G. Electrophile, substrate functionality, and catalyst effects in the synthesis of a-mono and disubstituted benzylamines via visible-light photoredox catalysis in flow. ChemCatChem 10, 3786-3794 (2018).

16. Leonard, K. A. et al. A selenopyrylium photosensitizer for photodynamic therapy related in structure to the antitumor agent aal with potent in vivo activity and no long-term skin photosensitization. J. Med. Chem. 43, 4488-4498 (2000)

17. Xu, H., Li, S., Huang, W., Guo, Q. \& Gao, Y. Synthesis and antitumor activities of erlotinib derivatives. J. Chin. Pharm. Univ. 39, 487-490 (2008).

18. Corma, A., Serna, P., Concepción, P. \& Calvino, J. J. Transforming nonselective into chemoselective metal catalysts for the hydrogenation of substituted nitroaromatics. J. Am. Chem. Soc. 130, 8748-8753 (2008).

19. Nørskov, J. K., Bligaard, T., Rossmeisl, J. \& Christensen, C. H. Towards the computational design of solid catalysts. Nat. Chem. 1, 37-46 (2009).

20. Bell, A. T. The impact of nanoscience on heterogeneous catalysis. Science 299, 1688-1691 (2003)

21. Chen, G. et al. Interfacial electronic effects control the reaction selectivity of platinum catalysts. Nat. Mater. 15, 564-569 (2016).

22. Mistry, H., Varela, A. S., Kühl, S., Strasser, P. \& Cuenya, B. R. Nanostructured electrocatalysts with tunable activity and selectivity. Nat. Rev. Mater. 1, 16009 (2016).

23. Yan, H. et al. Single-atom Pd1/graphene catalyst achieved by atomic layer deposition: remarkable performance in selective hydrogenation of 1,3butadiene. J. Am. Chem. Soc. 137, 10484-10487 (2015).

24. Schoenbaum, C. A., Schwartz, D. K. \& Medlin, J. W. Controlling the surface environment of heterogeneous catalysts using self-assembled monolayers. Acc. Chem. Res. 47, 1438-1445 (2014).

25. Liu, P., Qin, R., Fu, G. \& Zheng, N. Surface coordination chemistry of metal nanomaterials. J. Am. Chem. Soc. 139, 2122-2131 (2017).

26. Zhang, S. et al. Strong electronic metal-support interaction of $\mathrm{Pt} / \mathrm{CeO}_{2}$ enables efficient and selective hydrogenation of quinolines at room temperature. J. Catal. 359, 101-111 (2018)

27. Kattel, S., Ramírez, P. J., Chen, J. G., Rodriguez, J. A. \& Liu, P. Active sites for $\mathrm{CO}_{2}$ hydrogenation to methanol on $\mathrm{Cu} / \mathrm{ZnO}$ catalysts. Science 355, 1296-1299 (2017).

28. Xu, B., Yang, H., Zhou, G. \& Wang, X. Strong metal-support interaction in size-controlled monodisperse palladium-hematite nano-heterostructures during a liquid-solid heterogeneous catalysis. Sci. China Mater. 57, 34-41 (2014).

29. Matsubu, J. C. et al. Adsorbate-mediated strong metal-support interactions in oxide-supported Rh catalysts. Nat. Chem. 9, 120-127 (2016).

30. $\mathrm{Li}, \mathrm{Z}$. et al. Reactive metal-support interactions at moderate temperature in two-dimensional niobium-carbide-supported platinum catalysts. Nat. Catal. 1, 349-355 (2018)

31. Sibin, D., Rongming, W. \& Jingyue, L. Stability investigation of a high number density $\mathrm{Pt}_{1} / \mathrm{Fe}_{2} \mathrm{O}_{3}$ single-atom catalyst under different gas environments by HAADF-STEM. Nanotechnology 29, 204002 (2018).

32. Zhang, $\mathrm{P}$. et al. One-pot synthesis of ternary $\mathrm{Pt}-\mathrm{Ni}-\mathrm{Cu}$ nanocrystals with high catalytic performance. Chem. Mater. 27, 6402-6410 (2015).

33. Armbrüster, M. et al. Al13Fe4 as a low-cost alternative for palladium in heterogeneous hydrogenation. Nat. Mater. 11, 690-693 (2012).

34. Wu, Y. et al. Defect-dominated shape recovery of nanocrystals: a new strategy for trimetallic catalysts. J. Am. Chem. Soc. 135, 12220-12223 (2013).

35. Fu, Q.-Q., Li, H.-H., Ma, S.-Y., Hu, B.-C. \& Yu, S.-H. A mixed-solvent route to unique $\mathrm{PtAuCu}$ ternary nanotubes templated from $\mathrm{Cu}$ nanowires as efficient dual electrocatalysts. Sci. China Mater. 59, 112-121 (2016). 
36. Fan, H., Cheng, M., Wang, Z. \& Wang, R. Layer-controlled Pt-Ni porous nanobowls with enhanced electrocatalytic performance. Nano Res. 10, 187-198 (2017).

37. Zhao, E. W. et al. Silica-encapsulated Pt-Sn intermetallic nanoparticles: a robust catalytic platform for parahydrogen-induced polarization of gases and liquids. Angew. Chem. 129, 3983-3987 (2017).

38. Qi, Z. et al. Sub-4 nm PtZn intermetallic nanoparticles for enhanced mass and specific activities in catalytic electrooxidation reaction. J. Am. Chem. Soc. 139, 4762-4768 (2017).

39. Armbrüster, M. et al. Pd-Ga intermetallic compounds as highly selective semihydrogenation catalysts. J. Am. Chem. Soc. 132, 14745-14747 (2010)

40. Shao, L. et al. Nanosizing intermetallic compounds onto carbon nanotubes: active and selective hydrogenation catalysts. Angew. Chem. Int. Ed. 50, 10231-10235 (2011).

41. Friedrich, M., Teschner, D., Knop-Gericke, A. \& Armbrüster, M. Surface and subsurface dynamics of the intermetallic compound $\mathrm{ZnNi}$ in methanol steam reforming. J. Phys. Chem. C 116, 14930-14935 (2012).

42. Kang, Y., Pyo, J. B., Ye, X., Gordon, T. R. \& Murray, C. B. Synthesis, shape control, and methanol electro-oxidation properties of $\mathrm{Pt}-\mathrm{Zn}$ alloy and $\mathrm{Pt}_{3} \mathrm{Zn}$ intermetallic nanocrystals. ACS Nano 6, 5642-5647 (2012).

43. Jana, S., Chang, J. W. \& Rioux, R. M. Synthesis and modeling of hollow intermetallic Ni-Zn nanoparticles formed by the Kirkendall effect. Nano Lett. 13, 3618-3625 (2013).

44. Furukawa, S., Yoshida, Y. \& Komatsu, T. Chemoselective hydrogenation of nitrostyrene to aminostyrene over Pd- and Rh-based intermetallic compounds. ACS Catal. 4, 1441-1450 (2014).

45. Fouad, O. A., Ismail, A. A., Zaki, Z. I. \& Mohamed, R. M. Zinc oxide thin films prepared by thermal evaporation deposition and its photocatalytic activity. Appl. Catal. B 62, 144-149 (2006).

46. Camacho-Bunquin, J. et al. Atomically precise strategy to a PtZn alloy nanocluster catalyst for the deep dehydrogenation of n-butane to 1,3 butadiene. ACS Catal. 8, 10058-10063 (2018).

47. Boccuzzi, F., Chiorino, A. \& Guglielminotti, E. Effects of structural defects and alloying on the FTIR spectra of CO adsorbed on Pt/ZnO. Surf. Sci. 368, 264-269 (1998).

48. Brandt, R. K., Hughes, M. R., Bourget, L. P., Truszkowska, K. \& Greenler, R. $\mathrm{G}$. The interpretation of $\mathrm{CO}$ adsorbed on $\mathrm{Pt} / \mathrm{SiO}_{2}$ of two different particle-size distributions. Surf. Sci. 286, 15-25 (1993).

49. Brandt, R. K., Sorbello, R. S. \& Greenler, R. G. Site-specific, coupledharmonic-oscillator model of carbon monoxide adsorbed on extended, singlecrystal surfaces and on small crystals of platinum. Surf. Sci. 271, 605-615 (1992).

50. Yoshida, H., Narisawa, S., Fujita, S. I., Ruixia, L. \& Arai, M. In situ FTIR study on the formation and adsorption of $\mathrm{CO}$ on alumina-supported noble metal catalysts from $\mathrm{H}_{2}$ and $\mathrm{CO}_{2}$ in the presence of water vapor at high pressuresw. Phys. Chem. Chem. Phys. 14, 4724-4733 (2012).

51. Yoshida, H., Igarashi, N., Fujita, S. I., Panpranot, J. \& Arai, M. Influence of crystallite size of $\mathrm{TiO}_{2}$ supports on the activity of dispersed Pt catalysts in liquid-phase selective hydrogenation of 3-nitrostyrene, nitrobenzene, and styrene. Catal. Lett. 145, 606-611 (2015).

52. Ding, K. et al. Identification of active sites in $\mathrm{CO}$ oxidation and water-gas shift over supported Pt catalysts. Science 350, 189-192 (2015).
53. Cheng, B. \& Samulski, E. T. Hydrothermal synthesis of one-dimensional ZnO nanostructures with different aspect ratios. Chem. Commun. 0, 986-987 (2004).

\section{Acknowledgements}

This work was supported by China Ministry of Science and Technology under Contract of 2016YFA (0202801), the National Natural Science Foundation of China (21521091, 21390393, U1463202, 21471089, 21671117, 21703219, 21521005), and 111 Project (B16028).

\section{Author contributions}

A.H. and J.Z. performed the experiments, collected and analyzed the data, and wrote the paper. W.S. conducted the density functional theory calculation and analysis. S.Z, Y.H and Q.F assisted in HR-TEM, STEM and EDX elemental mapping characterizations. W.C. and L.Z. helped with XANES and EXAFS spectrometry analyses. L.G. assisted in the AC HAADF-STEM characterization. C.C. and Q.P. helped with data analyses and discussions. D.W. and Y.L. conceived the experiments, planned synthesis, analyzed results and wrote the paper.

\section{Additional information}

Supplementary Information accompanies this paper at https://doi.org/10.1038/s41467019-11794-6.

Competing interests: The authors declare no competing interests.

Reprints and permission information is available online at http://npg.nature.com/ reprintsandpermissions/

Peer review information: Nature Communications thanks the anonymous reviewers for their contribution to the peer review of this work. Peer reviewer reports are available.

Publisher's note: Springer Nature remains neutral with regard to jurisdictional claims in published maps and institutional affiliations.

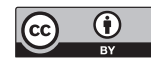

Open Access This article is licensed under a Creative Commons Attribution 4.0 International License, which permits use, sharing, adaptation, distribution and reproduction in any medium or format, as long as you give appropriate credit to the original author(s) and the source, provide a link to the Creative Commons license, and indicate if changes were made. The images or other third party material in this article are included in the article's Creative Commons license, unless indicated otherwise in a credit line to the material. If material is not included in the article's Creative Commons license and your intended use is not permitted by statutory regulation or exceeds the permitted use, you will need to obtain permission directly from the copyright holder. To view a copy of this license, visit http://creativecommons.org/ licenses/by/4.0/.

(C) The Author(s) 2019 